\section{BASIC-PLUS-2 programs to perform experimental procedures for paired associate verbal learning}

\section{JANUSZ PREZEOREK, RUBEN SANCHEZ, and RICHARD DENI \\ Rider College, Lawrenceville, New Jersey 08648}

These programs were designed to demonstrate standard methods for paired associate learning. They are also suitable for use in experiments in which individual subjects are exposed to the material to be learned and tested at a video computer terminal. The programs are written in BASIC-PLUS-2 for the Digital Equipment Corporation DECsystem 2060 computer under the Tops-20 operating system. The four programs in the package include (1) FILEMAKER, used to create input files holding the paired associates to be learned, (2) ANTICIPATION, used to present paired associates and record learning progress with the standard method of anticipation, (3) DISPLAY, used to present paired associates under controlled conditions and specifications, and (4) RECOGNITION, used to present a multiplechoice recognition test procedure for paired associates learned during a run of DISPLAY.

\section{General Features}

Input. The experimenter enters individual paired associates via the keyboard, prior to testing a subject. During testing, lists of paired associates stored in files are accessed by the test programs and displayed to subjects. Subjects report via the keyboard, either by entering a missing response term or by selecting a multiple-choice option.

Timing. The test programs use the BASIC-PLUS-2 TIME(0) function to read the value of a system clock. This clock can operate only with a $1-\mathrm{sec}$ time base, but that is adequate for demonstration purposes. The clock begins incrementing from zero at 12:00 midnight.

Output. The test programs create output files under file names specified by the experimenter. All data recorded on the subject's performance during learning or testing are printed into these files.

\section{Specific Features}

FILEMAKER Program. This program creates input files of paired associates for later use in conjunction with the three learning/testing programs. Input files are formatted as virtual core files for simplicity of access. Redimensioning virtual core arrays is a user option; however, all virtual core file DIM statements in any of the four programs must be identical. FILEMAKER must be run to create each paired associate list to be used in the experiment (for example, if type of list represented a level of an independent variable). Any character string can be input as either the stimulus or the response term in a paired associate. In addition, the experimenter must designate the number of pairs in the list and assign a specific file name to the list.

ANTICIPATION Program. This program performs the standard method of anticipation learning procedure for paired associates. Several parameters must be input by the experimenter prior to testing a subject. These include (1) the name of the input file holding the paired associate list, (2) the name of the output file to be created, (3) the duration of the intertrial interval, (4) the duration of the stimulus-term/response-term interval, and (5) the learning criterion (all or some portion of the response terms in the list anticipated correctly).

During the run of this program, stimulus terms are chosen from the list at random and displayed for a fixed amount of time. Each stimulus term is displayed at the center of the screen, using Digital Equipment Corporation VT-52 terminal escape code sequences to move the cursor. If the subject can respond with a response term, he does so by typing it at the keyboard. If the response is either correct or incorrect, the response term is displayed on the screen for a fixed amount of time and the trial is completed. If the subject has no response to make, he presses the return key; the correct response term is displayed, and the trial is completed. Before the next trial begins, performance factors are recorded, including the latency to make a response and whether the response was correct. Trials continue in this manner until one complete cycle of the list can be displayed with the correct number of anticipations specified by the criterion.

The ANTICIPATION program can also be used to carry out a free recall test in which only stimulus terms are presented and response terms are never displayed by the program after subject errors or failures to respond. For this application, the DISPLAY program could be used to expose subjects to pairs prior to testing with the ANTICIPATION program.

DISPLAY Program. This program displays paired associates on the screen, one pair at a time, with stimulus and response terms shown side by side at the center of the screen. The parameters that must be specified by the experimenter include (1) the name of the input file, (2) whether stimulus terms are to be displayed in random order or in list order, and (3) the intertrial interval. The subject makes no response during a run of DISPLAY but is simply exposed to a list of paired associates.

RECOGNITION Program. This program is used following a run of the DISPLAY program and carries out a multiple-choice recognition test. The parameters specified by the experimenter prior to testing are (1) name of the input file, (2) name of the output file, 
and (3) intertrial interval. During the program run, each stimulus term in the list of paired associates is presented near the center of the screen. Just to the right are listed three possible response choices. The position of the correct response term among the three choices is random. The two incorrect alternatives are other response terms from the list. The subject must respond at the keyboard by entering the number of the correct choice. The screen is cleared after each trial. The performance factors recorded include the latency to respond and whether the response was correct. Each stimulus term is selected at random, and trials continue until all stimulus terms have been displayed.

The data printed for each subject include the following: (1) trial-by-trial record of latency and correct/ incorrect, (2) mean latencies for correct, incorrect, and overall, (3) mean trials correct/incorrect, and (4) percent trials correct/incorrect. These data are printed in table form in the output file specified by the experimenter.

Command File Execution Feature. The TOPS-20 operating system for the DECsystem 20 computers allows the user to create a file consisting of a series of commands to be executed as if they were input from the keyboard at the time of a program run. This feature allows an experimenter to preset any configuration of run commands to carry out a complete series of steps with no experimenter interruption once the display/ testing has begun. For example, in order to use this program package to carry out a retroactive interference procedure, the experimenter would run FILEMAKER to create two paired associate lists. List 1 would contain A-B pairs. List 2 would contain A-C pairs. Second, three copies of the ANTICIPATION program would be created to present the three stages in the procedure (input statements would have to be removed in these copies and replaced with "let" statements, setting constant values for file names and parameters). Next, the experimenter would create a command file using the screen editor. These commands would consist of entering BASIC mode, running ANTICIPATION (Copy 1), with the A-B list file name and other program parameters set as constants in the program, running ANTICIPATION (Copy 2), with the A-C list file name and other parameters as constants, and running ANTICIPATION (Copy 3), with the A-B list file name and parameters as constants. This command file would then be executed with a single command typed by the experimenter before each subject is tested. The full series of procedures would then be executed as specified.

\section{Transportability}

The programs are written in the Digital Equipment Corporation's BASIC-PLUS-2 language for the DECsystem 20 computers under the Tops- 20 operating system. Because the programs use "virtual core" arrays, transportability is limited. However, Digital Equipment Corporation VAX systems with BASIC-PLUS can also run the programs. Within each of the programs in this package, alternate statements, when required for the VAX, are shown in REM lines.

\section{Availability}

The program listings are available at no charge from Richard Deni, Psychology Department, Rider College, Lawrenceville, New Jersey 08648.

(Accepted for publication December 30, 1982.) 